\title{
28 Research Square \\ 2019 Novel Coronavirus (COVID-19) pneumonia: an unusual lung ultrasound presentation.
}

\section{Letizia Consoli ( $\sim$ letizia.consoli@poliambulanza.it)}

Department of Emergency Medicine, Fondazione Poliambulanza, Brescia, Italy.

\section{Vittorio Bendotti}

Department of Emergency Medicine, Fondazione Poliambulanza, Brescia, Italy

\section{Sara Cicchinelli}

Department of Emergency Medicine, Fondazione Poliambulanza, Brescia, Italy. Department of

Emergency Medicine, Fondazione Policlinico A. Gemelli IRCCS, Rome, Italy

\section{Federico Gaioni}

Department of Emergency Medicine, Fondazione Poliambulanza, Brescia, Italy

\section{Paola Prandolini}

Department of Radiology and Medical Imaging, Fondazione Poliambulanza, Brescia, Italy

\section{Monica Bettonagli}

Department of Emergency Medicine, Fondazione Poliambulanza, Brescia, Italy

\section{Paolo Terragnoli}

Department of Emergency Medicine, Fondazione Poliambulanza, Brescia, Italy

\section{Case Report}

Keywords: Covid-19, Pneumonia, Pneumothorax, Lung Ultrasound

Posted Date: April 28th, 2020

DOI: https://doi.org/10.21203/rs.3.rs-25490/v1

License: (c) (1) This work is licensed under a Creative Commons Attribution 4.0 International License.

Read Full License 


\section{Abstract}

In December 2019, a novel coronavirus (SARS-Cov-2) was first reported in Wuhan, China, and rapidly spread around the world, leading to an international emerging public health emergency. As reported from Chinese experiences, approximately $20 \%$ of patients had a severe course, requiring intensive care, with an overall case fatality rate of $2.3 \%$. In diagnosis, chest computed tomography most commonly showed ground-glass opacity with or without consolidative patterns.

Herein we report a case of a patient affected by COVID-19 pneumonia referred in the emergency department of our institution on 4 April 2020 with peculiar lung ultrasound findings.

\section{Introduction}

The outbreak of an atypical pneumonia was first reported in Wuhan city, the capital of Hubei province in China, on December 2019 [1]. In January 2020, Chinese scientists isolated a novel coronavirus from patients affected by viral pneumonia, denominated severe acute respiratory syndrome coronavirus 2 (SARS-COV-2) and, in February 2020, the World Health Organization designated as COVID-19, the coronavirus disease caused by SARS-COV-2.

As recently depicted in a report from the Chinese Center of Disease control on 44500 SARS-COV-2 patients, severe respiratory symptoms were reported in $14 \%$ of cases, characterized by dyspnea, hypoxia, or $>50 \%$ lung involvement on imaging. $5 \%$ of patients were critical (respiratory failure, shock, or multiorgan system dysfunction). In this study, all deaths occurred among patients with critical illness and the overall case fatality rate was $2.3 \%$. The case fatality rate among patients with critical disease was $49 \%$ [2]. The most common complications observed in severe cases included acute respiratory distress syndrome and respiratory failure while less common complications included secondary infection, acute cardiac injury, and hypoxic encephalopathy, acute kidney injury, shock and acute liver injury [3-6]. Oropharyngeal and nasopharyngeal tract swabs need to be tested to confirm a clinical suspect of SARSCOV-2 infection [1]. Moreover, chest radiography and computed tomography (CT) scan complete the diagnostic approach to COVID-19 patients, usually showing ground-glass opacities, sometimes associated to consolidative patterns $[7,8,9]$. In addition to these primary imaging methods, lung ultrasound (LUS) provide a timely bedside evaluation of COVID-19 patients, both in the primary assessment and during monitoring to adjust treatment plan [10].

\section{Case Report}

A 37-year-old male, without known previous comorbidities, presented at the Emergency Department of Fondazione Poliambulanza Hospital (a middle size general private hospital in Brescia, northern Italy), complaining fever and cough for 2 weeks. Moreover, he reported progressive dyspnea, limiting his activities of daily living. 
Peripheral blood examinations showed a mild lymphopenia $(750 / \mathrm{mmc})$ and an increased C-reactive protein $(10 \mathrm{mg} / \mathrm{L})$. Physical examination revealed a body temperature $38^{\circ} \mathrm{C}$, respiratory rate of 30 breath per minutes, blood oxygen saturation $90 \%$ on room air. Arterial blood gas test revealed a moderate hypoxemia (arterial pressure oxygen- $\mathrm{PaO} 2=50 \mathrm{mmHg}$ ) and mild hypocapnia $(\mathrm{PaCO} 2=30 \mathrm{mmHg}$ ). Nasopharyngeal swab specimen was collected for testing SARS-COV-2 and polymerase chain reaction revealed positive viral nuclear acid in the sample. A primary bedside lung ultrasound (LUS) assessment was immediately performed in order to provide a real-time estimate of COVID-19 lung involvement. The scan performed with convex array probe showed multifocal and confluent B-lines with thickening of the pleural line at the medium right field (fig. 1) and a dynamic air bronchogram sign at the posterior homolateral lower field (fig. 2). On the left side, the LUS showed no pleural sliding nor lung point sign (fig. 3). Chest X-ray confirmed a massive pneumothorax of the left lung and interstitial involvement of the right one (fig. 4). A chest tube was immediately placed, and a subsequent CT-scan confirmed the lung reexpansion, bilateral consolidations with CT scan-score of 25-50\% (fig. 5), according to semi-quantitative method made by Chinese group [5]. During the hospitalization high flow nasal cannula oxygen therapy, steroid therapy without antiviral drugs, considering the long-lasting symptoms, were delivered. Final chest $\mathrm{x}$-ray before the discharge showed a significant improvement and the patient, until now, is asymptomatic with no need of therapy.

\section{Discussion}

COVID-19 has been previously described as a high rate infection disease with several systemic complications $[2,5,6]$. Even though chest $\mathrm{X}$-ray and CT-scans are widely used in the primary instrumental assessment of COVID-19 patients $[7,8,9]$, emerging evidences have explored the role of ultrasound in the diagnosis and treatment $[10,11,12]$. Frequent abnormal ultrasound imaging findings such as B-lines, consolidation areas or alteration of the pleural line have been recently characterized [12]. On the other hand, ultrasound may produce a real-time and dynamic evaluation, even in cases with critical complications of severe COVID-19 pneumonia, such as pneumothorax.

As described in the present report, COVID-19 infection, displaying its lung tropism, may be associated to multiple and diffuse lesions. Our data are consistent to those recently published by Sun et al [13], who explored the outcome of a patient with mediastinal emphysema and pneumothorax. As detailed by Sun et al, pneumothorax could be produced as a consequence of a sudden increase of the alveolar pressure into the pneumonic consolidations [13]. Lung compliance is high compared with other etiologies of ARDS and the rate of barotrauma appears to be low with only $2 \%$ developing pneumothorax compared with $25 \%$ of those with SARS severe acute respiratory syndrome $[5,6]$. Accordingly, the alveolar rupture of the patient here described was localized at the consolidated area, as revealed by the CT-scan. Along this line, lung ultrasonography could be performed at patient's bedside and it could be considered as primary, handy tool to quickly assess and subsequently treat the condition. The integration of ultrasound images with CT images may be effective in the activation of a comprehensive management plan. 
In conclusion, this report reminds us of the importance of consider the possibility of lung complications during COVID-19 infections, such as pneumothorax, even in subjects with no history of previous similar events nor predisposing risk factors. In addition, the appearance of these pathological findings and the severity of interstitial lung involvement are not commonly closely related.

This report also highlights the contribute of lung ultrasound to guarantee the appropriate identification and follow-up. We also speculate, according to the pathogenetic mechanism of viral primum movens and known subsequent excessive immune response, that early introduction of antiviral drugs associated with chloroquine and corticosteroids in patients with symptoms suspicious of COVID-19 disease help not only to treat the infection but also to prevent the complications onset. However, more direct observation is needed to confirm this latter assumption.

\section{Declarations}

\section{Funding}

This research received no external funding.

\section{Conflicts of Interest}

The authors declare no conflict of interest.

\section{Informed consent}

A written informed consent for publication was obtained from the individual involved in the study

\section{References}

1. Guan WJ, Ni ZY, Hu Y,Liang WH, Ou CQ, He JX, Liu L, Shan H, Lei CL, Hui DSC, Du B, Li LJ, Zeng G, Yuen KY, Chen RC, Tang CL, Wang T, Chen PY, Xiang J, Li SY, Wang JL, Liang ZJ, Peng YX, Wei L, Liu Y, Hu YH, Peng P, Wang JM, Liu JY, Chen Z, Li G, Zheng ZJ, Qiu SQ, Luo J, Ye CJ, Zhu SY (2020) Clinical Characteristics of Coronavirus Disease 2019 in China. N Engl J Med, Feb 28. doi: 10.1056/NEJMoa2002032

2. Wu Z, McGoogan JM (2020) Characteristics of and Important Lessons From the Coronavirus Disease 2019 (COVID-19) Outbreak in China: Summary of a Report of 72314 Cases From the Chinese Center for Disease Control and Prevention. Jama, Feb 24. doi: 10.1001/jama.2020.2648.

3. Chen G,Wu D, Guo W, Cao Y, Huang D, Wang H, Wang T, Zhang X, Chen H, Yu H, Zhang X, Zhang M, Wu S, Song J, Chen T, Han M, Li S, Luo X, Zhao J, Ning Q (2020) Clinical and immunologic features in severe and moderate Coronavirus Disease 2019. J Clin Invest, Apr 13. doi:

$10.1172 / \mathrm{JCl} 137244$. 
4. Xu Z, Shi L, Wang Y, Zhang J, Huang L, Zhang C, Liu S,Zhao P, Liu H, Zhu L, Tai Y, Bai C, Gao T, Song J, Xia P, Dong J, Zhao J, Wang FS (2020) Pathological findings of COVID-19 associated with acute respiratory distress syndrome. Lancet Respir Med; 8 (4): 420-422. doi: 10.1016/S22132600(20)30076-X.

5. Gomersall CD, Joynt GM, Lam P, Li T, Yap F, Lam D, Buckley TA, Sung JJ, Hui DS, Antonio GE, Ahuja AT, Leung P (2004) Short-term outcome of critically ill patients with severe acute respiratory syndrome. Intensive Care Med, 30(3):381-7. doi: 1007/s00134-003-2143-y

6. Yang X, Yu X, Xu J, Shu H, Xia J, Liu H, Wu Y, Zhang L, Yu Z, Fang M, Yu T, Wang Y, Pan S, Zou X, Yuan S Shang Y (2020) Clinical course and outcomes of critically ill patients with SARS-CoV-2 pneumonia in Wuhan, China: a single centered, retrospective, observational study. Lancet Respirat Med, Feb 24. doi: 10.1016/S2213-2600(20)30079-5.

7. Ye Z,Zhang Y, Wang Y, Huang Z, Song B (2020) Chest CT manifestations of new coronavirus disease 2019 (COVID-19): a pictorial review. Eur Radiol, Mar 19. doi: 10.1007/s00330-020-06801-0.

8. Xingzhi Xie, Zheng Zhong, Wei Zhao, Chao Zheng, Fei Wang, Jun Liu (2020) Chest CT for typical 2019-nCoV Pneumonia: Relationship to negative RT-PCR testing. Radiology, Feb 12. doi:

10.1148/radiol.2020200343.

9. Zhao X,Liu B, Yu Y, Wang X, Du Y, Gu J, Wu X (2020) The characteristics and clinical value of chest CT images of novel coronavirus pneumonia. Clin Radiol, 75 (5): 335-340. doi:

10.1016/j.crad.2020.03.002.

10. Vetrugno L, Bove T, Orso D, Barbariol F, Bassi F, Boero E, Ferrari G, Kong R. (2020) Our Italian Experience Using Lung Ultrasound for Identification, Grading and Serial Follow-up of Severity of Lung Involvement for Management of Patients with COVID- Echocardiography, Apr 1. doi: 10.1111/echo. 14664

11. Sikachi R,Agrawal A (2020) Whole body point of care ultrasound for COVID-19: a multi-system approach to a multi-system disease. Anaesthesia, Apr 16. doi: 10.1111/anae.15087

12. Sofia S,Boccatonda A, Montanari M, Spampinato M, D'ardes D, Cocco G, Accogli E, Cipollone F, Schiavone C (2020) Thoracic ultrasound and SARS-COVID-19: a pictorial essay. J Ultrasound, Apr 16. doi: 10.1007/s40477-020-00458-7.

13. Sun R, Liu H, Wang (2020) Mediastinal Emphysema, Giant Bulla, and Pneumothorax Developed during the Course of COVID-19 Pneumonia. Korean J Radiol, 21: e43. doi: 3348/kjr.2020.0180.

\section{Figures}




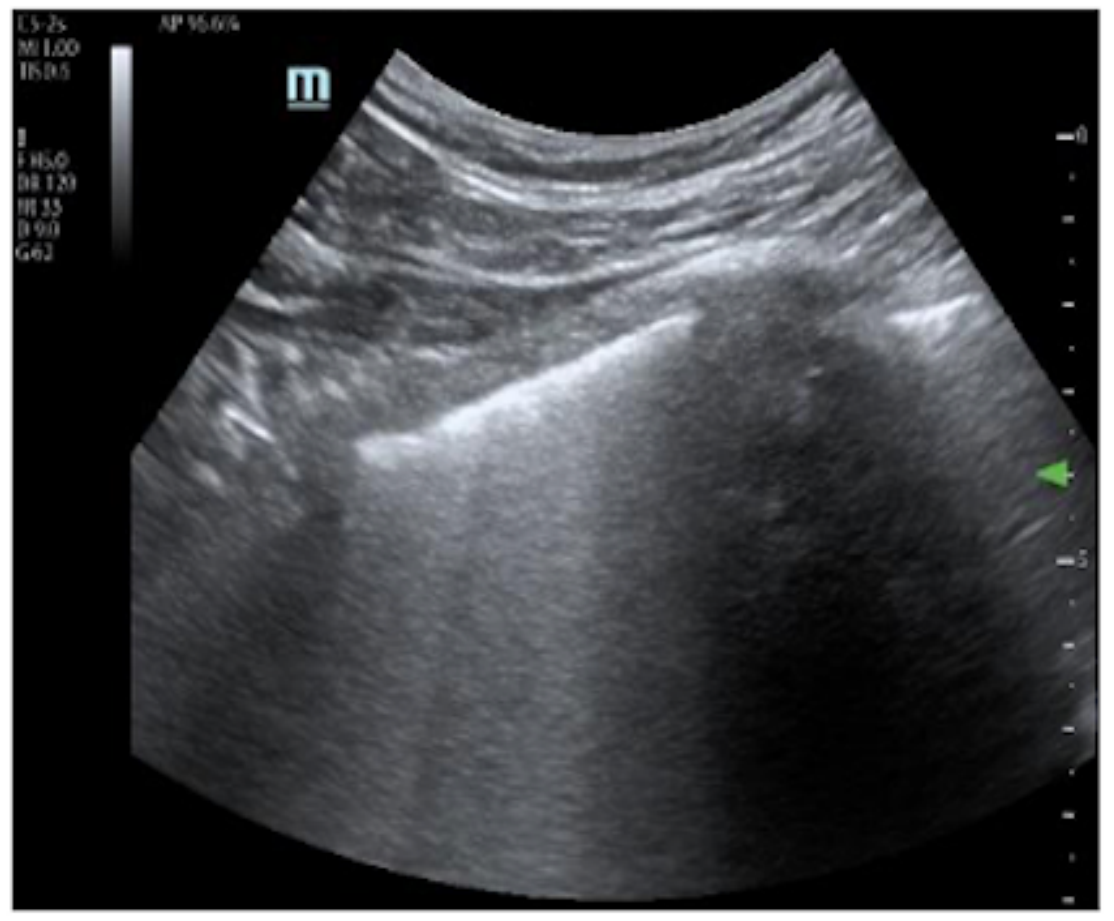

\section{Figure 1}

Convex array probe showed multifocal and confluent B-lines with thickening of the pleural line in the medium field of the right side. 


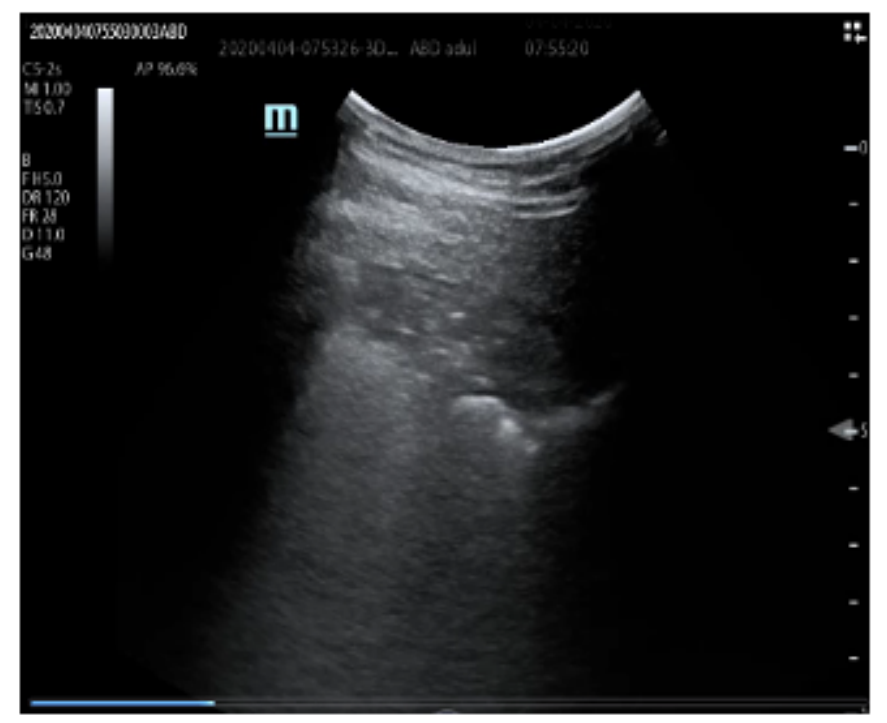

Figure 2

Convex array probe showed consolidation with dynamic air bronchogram in inferior field of the right lung. 


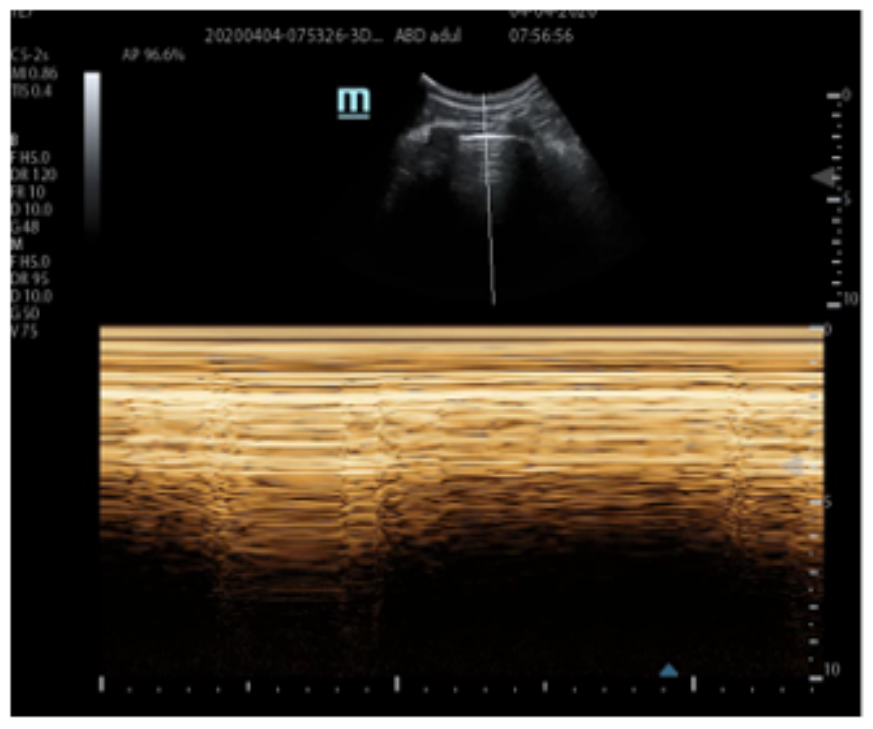

Figure 3

Convex array probe showed the absence pleural sliding at the left lung with a "barcode sign" at the Mmode evaluation. 


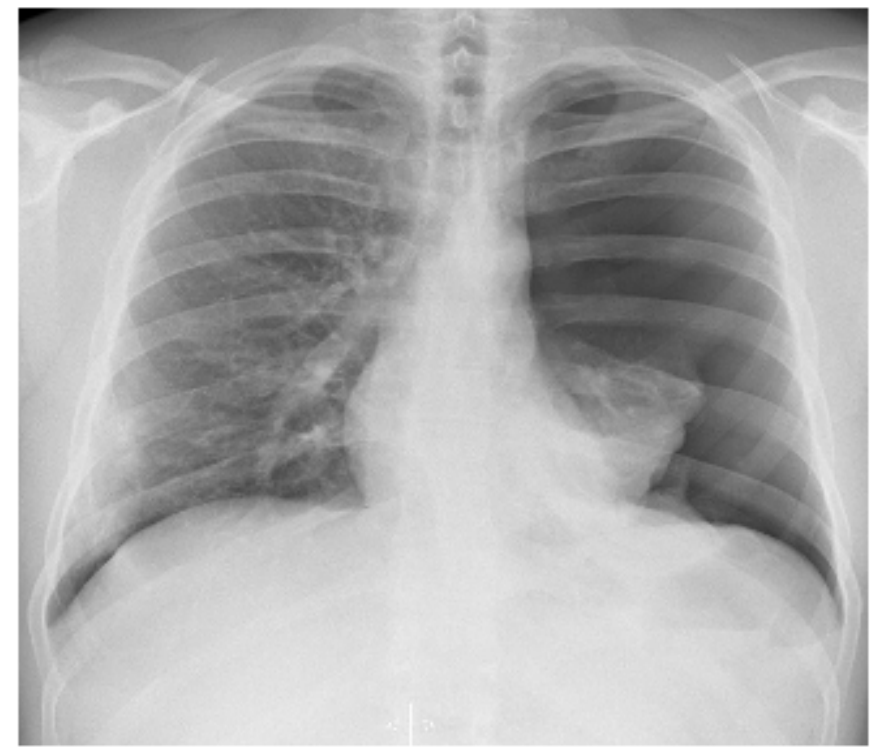

Figure 4

Chest X-ray showed complete left pneumothorax and right interstitial involvement. 


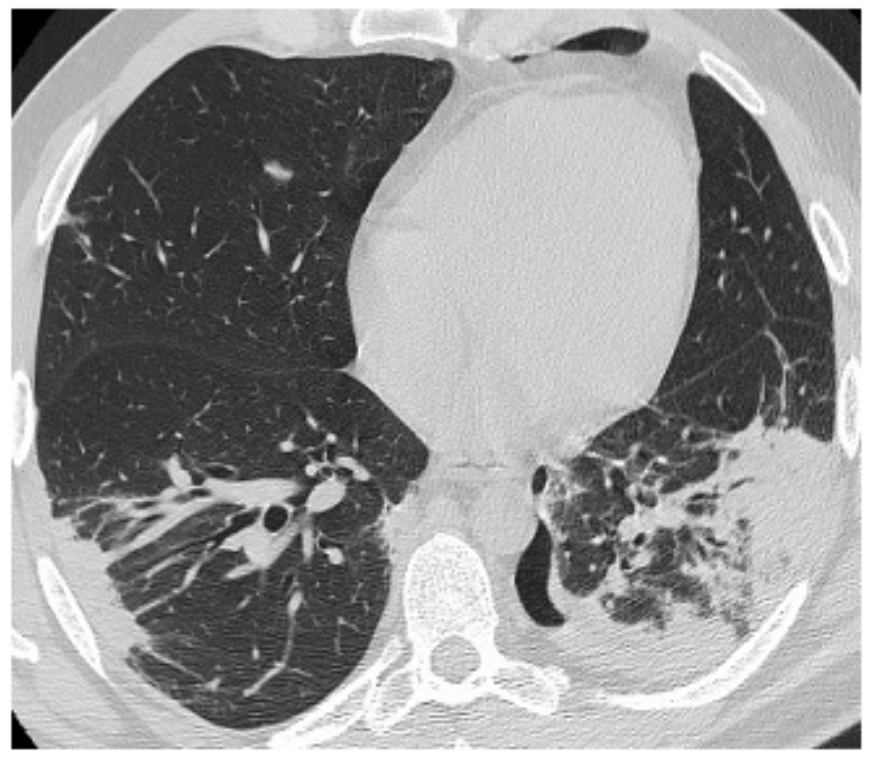

Figure 5

CT-scan performed after chest tube placement showed bilateral consolidations, mainly at the posterior lower field of the left lung. 